\title{
The multi-purse string maze procedure: A new surgical technique to perform the full maze procedure without atriotomies
}

Niv Ad, MD

From the Inova Heart and Vascular Institute, Falls Church, Va.

Niv Ad reports lecture fees from Medtronic.

Received for publication Jan 15, 2007; revisions received March 6, 2007; accepted for publication April 12, 2007.

Address for reprints: Niv Ad, MD, Inova Heart and Vascular Institute, 3300 Gallows Rd, Falls Church, VA 22042 (E-mail: niv. ad@inova.com).

J Thorac Cardiovasc Surg 2007;134:717-22

$0022-5223 / \$ 32.00$

Copyright (C) 2007 by The American Association for Thoracic Surgery

doi:10.1016/j.jtcvs.2007.04.043
Objective: The maze procedure is the most effective surgical procedure for atrial fibrillation. In recent years, a number of different surgical ablation devices were introduced and gradually replaced the need for the procedure to be performed by using the cut-and-sew technique. In this article a new surgical approach is presented using a full maze IV lesion set, without atriotomies.

Methods: Between January 2005 and June 2006, 12 patients underwent operations with the multi-purse string technique: 9 patients as a combined procedure with coronary artery bypass grafting, 2 patients as a combined procedure with aortic valve replacement, and 1 patient as a standalone procedure. In 5 additional patients, a small left atrial atriotomy was required to ensure the completeness of the mitral valve isthmus lesion. A combination of bipolar radiofrequency and cryothermal energies was used in all cases. All patients were part of our comprehensive follow-up and local atrial fibrillation registry.

Results: No perioperative morality, cerebrovascular accidents/transient ischemic attacks, or both were documented in this series. In a mean follow-up of $13 \pm 6$ months, 1 late death was documented, and $91 \%$ of the patients are free from atrial fibrillation or flutter.

Conclusions: Our experience suggests that the maze IV procedure can be performed without the need for atriotomies by using the multi-purse string approach. The intermediate results are promising, with high rates of success in ablating atrial fibrillation. Future studies should be performed to validate the safety of the use of bipolar radiofrequency devices endocardially on a beating heart.

7 he maze procedure has proved to be the most effective surgical procedure to treat atrial fibrillation. ${ }^{1,2}$ Since 1987, the maze I procedure has been modified a few times to improve sinus node function and interatrial transport. ${ }^{3}$ It was not until 1999 that a complete maze III procedure was performed without the use of multiple atriotomies; cryoablation technology creating linear cryoablation lines was used instead. ${ }^{4}$ Since then, a few ablation devices using various energy sources have been introduced to surgeons, and most have proved to be very safe and effective., When using the ablation devices, the atrial incisions of the standard maze procedure are replaced by linear ablation lesions. ${ }^{7}$ Whether surgical incisions or ablation lesions are used to perform the maze procedure, it is essential to create transmural atrial lesions to ensure a high success rate.,

One of the challenges we are facing in recent years is the need to minimize the time required to perform the maze procedure with a goal of eliminating the need for the use of cardiopulmonary bypass and applying minimally invasive approaches, especially when surgically treating those patients with lone atrial fibrillation.

In the current report, I introduce a new surgical approach using multiple epicardial purse-string sutures on both the right and left atrial epicardial surfaces. By using 


\section{Abbreviations and Acronyms \\ AVR = aortic valve replacement \\ $\mathrm{CABG}=$ coronary artery bypass grafting \\ IVC $=$ inferior vena cava}

this technique, a full maze IV lesion set can be applied without the need for atriotomies. ${ }^{7}$ This technique can be used in patients with lone atrial fibrillation or in those patients with atrial fibrillation with an indication for aortic valve replacement (AVR), coronary artery bypass grafting $(\mathrm{CABG})$, or both in whom it is not required to approach the left atrium through an atriotomy otherwise.

\section{Materials and Methods}

Between January 2005 and June 2006, 12 patients underwent the multi-purse string maze procedure (Table 1). In 5 additional patients the procedure was modified because of the need for a small left atrial atriotomy to guarantee a reliable left atrial isthmus ablation.

\section{Surgical Procedure}

In this approach the atrial incisions of the standard maze procedure are replaced by linear lesions that are applied with either bipolar radiofrequency energy or cryogenic ablative energy. Whether surgical incisions, cryolesions, or radiofrequency are used to perform the maze procedure, it is essential to create transmural atrial lesions to prevent late recurrence of atrial fibrillation.

\section{Operative Steps}

The multi-purse string maze procedure is performed through a standard median sternotomy. A variety of cryoprobes are used, the most important being a rigid straight probe that can create linear cryolesions 5 to $7 \mathrm{~cm}$ in length. A $15-\mathrm{mm}$ right angle probe is also

\section{Table 1. Demographics and clinical characteristics}

\begin{tabular}{lrc}
\hline & No. of Patients (\%) & Mean \pm SD \\
\hline Age (y) & & $62.7 \pm 7.4$ \\
$\begin{array}{l}\text { Sex (male) } \\
\text { Permanent atrial fibrillation }\end{array}$ & $10(83 \%)$ & \\
$\quad$ Duration (mo) & $3(25 \%)$ & \\
$\begin{array}{l}\text { Persistent atrial fibrillation } \\
\quad \text { Duration (mo) }\end{array}$ & $8(67 \%)$ & $68.6 \pm 22$ \\
$\begin{array}{l}\text { Paroxysmal trial Fibrillation } \\
\quad \text { Duration (mo) }\end{array}$ & $1(8 \%)$ & $69.9 \pm 75.7$ \\
$\begin{array}{l}\text { History of thromboembolic events } \\
\text { Left atrial size (cm) }\end{array}$ & $1(8 \%)$ & 11.8 \\
Maze only & $1(8 \%)$ & $4.6 \pm 0.8$ \\
Maze + CABG & $9(75 \%)$ & \\
Maze + AVR & $2(17 \%)$ & \\
\hline
\end{tabular}

$S D$, Standard deviation; $C A B G$, coronary artery bypass grafting; $A V R$, aortic valve replacement. helpful in most cases (Cooper Surgical). Special attention should be paid to the application of the cryolesions. In this technique cryothermal energy is used to create 3 different ablation lines, 2 in the right atrium and 1 across the mitral valve isthmus. The correct placement of the cryoprobes next to the mitral or tricuspid valve annulus can be done either by using echocardiographic guidance or manual palpation. The cryoprobe is then firmly attached to the endocardial surface, and the ablation process starts. Because the cryolesions actually can be observed as they develop, it is a simple matter to determine when transmural ablation has or has not occurred. Based on published data, cryoablation is maintained for 2 minutes after the appearance of the ice ball over the epicardial surface. $^{8}$

The bipolar radiofrequency devices that were used in this series were the Atricure Isolator for the first 6 cases and the Medtronic Cardioblate LP surgical ablation device.

Right atrial lesions. Creation of the right atrial lesions can be performed without the use of cardiopulmonary bypass but after full heparinization and cannulation of the aorta.

The first purse-string suture is placed in the posterior-lateral right atrium, and one jaw of a bipolar device is inserted through a stab wound created in the middle of the purse string into the inside of the right atrium so that one jaw of the device is positioned epicardially and the second endocardially. A lesion is placed in a cephalad direction into the posterolateral aspect of the superior vena caval orifice and away from the sinoatrial node (Figure 1, A). By using the same purse-string suture, a second lesion is placed into the orifice of the inferior vena cava (IVC) to complete the longitudinal lesion from the superior vena cava to the IVC (Figure 1, B). Through the same purse-string suture, a third lesion is made along the lateral wall of the right atrium down to the interatrial septum, and the right pulmonary vein is placed (Figure $1, C$, closed arrow). The first purse-string suture is tied, and a second purse-string suture is placed near the atrioventricular groove of the free wall of the right atrium. The bipolar device is then inserted through this second purse-string suture to create the linear $\mathrm{T}$ lesion across the lower right atrium (Figure 1, C). By using the same purse-string suture, a cryolesion is applied to extend the T lesion down to the level of the tricuspid valve annulus at the junction of the anterior and posterior commissures (Figure 1, $D$, right closed arrow). The second purse-string suture is then used to insert the coronary sinus catheter for retrograde administration of cardioplegia. A third purse-string suture is placed on the base of the right atrial appendage. A lateral right atrial cryolesion is placed from the tip of the atrial appendage toward the previously placed $\mathrm{T}$ lesion, leaving at least $3 \mathrm{~cm}$ between its tip and the T lesion. By using the same purse-string suture in the right atrial appendage, a cryolesion is placed from the appendage down to the anteromedial tricuspid valve annulus at the septal commissure (Figure 1, D, left closed black arrow).

With the last purse-string suture and after extending the incision to the right atrial appendage, the 2-stage venous cannula is introduced and secured.

Left atrial lesions. After the completion of the right atrial lesions, cardiopulmonary bypass is usually instituted, and the next few steps are performed before we crossclamp the aorta.

The interatrial groove is dissected completely, the right superior and right inferior pulmonary veins are dissected free circum- 

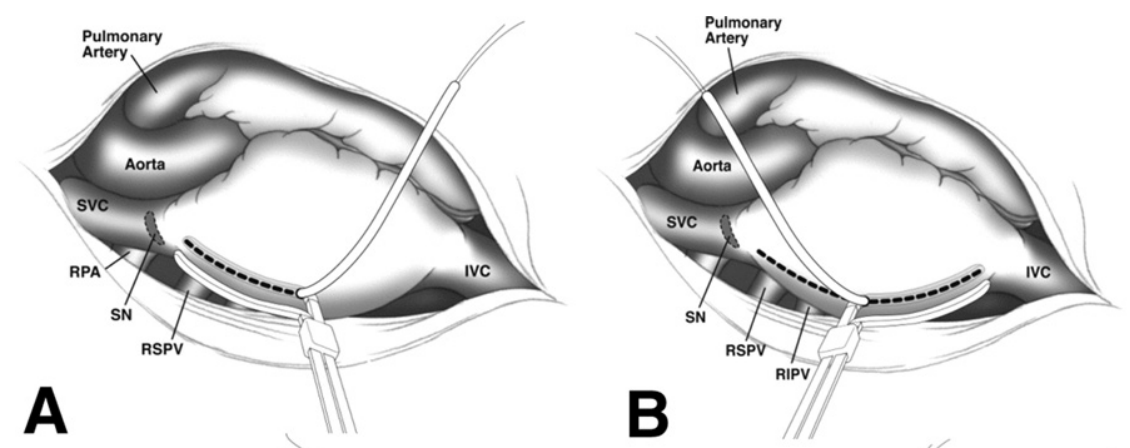

Figure 1. Right atrial lesion set. SVC, Superior vena cava; $S N$, sinus node; RSPV, right superior pulmonary vein; $R I P V$, right inferior pulmonary vein; IVC, inferior vena cava.

ferentially, and an umbilical tape is placed around the right pulmonary veins. The bipolar device is then placed around the right pulmonary veins. Multiple ablations are performed to ensure transmurality and conduction block (Figure 2, A).
The ventricular apex is then retracted in a cephalad direction out of the pericardium by using the left hand to expose the intrapericardial segments of both left pulmonary veins. After minimal dissection around the left pulmonary veins and placement of
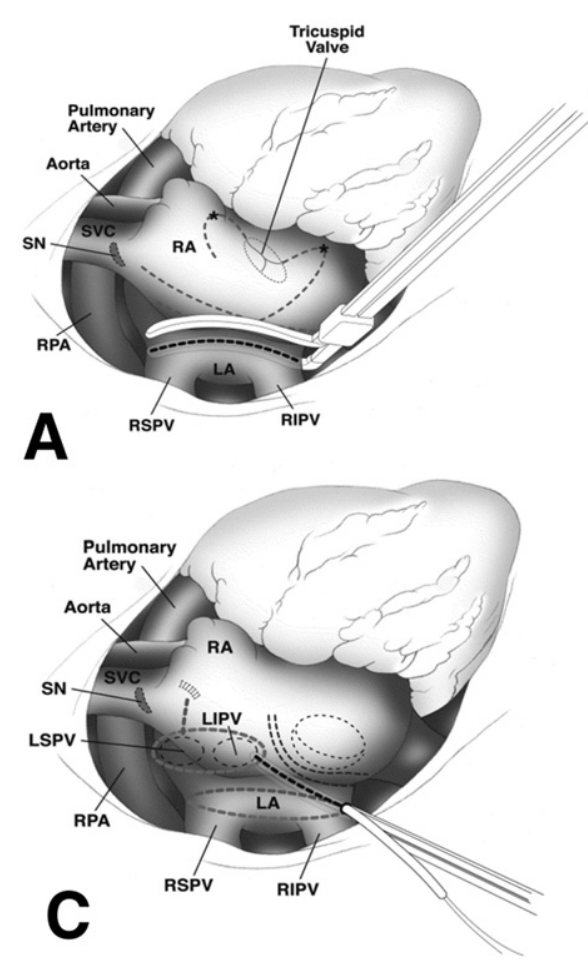

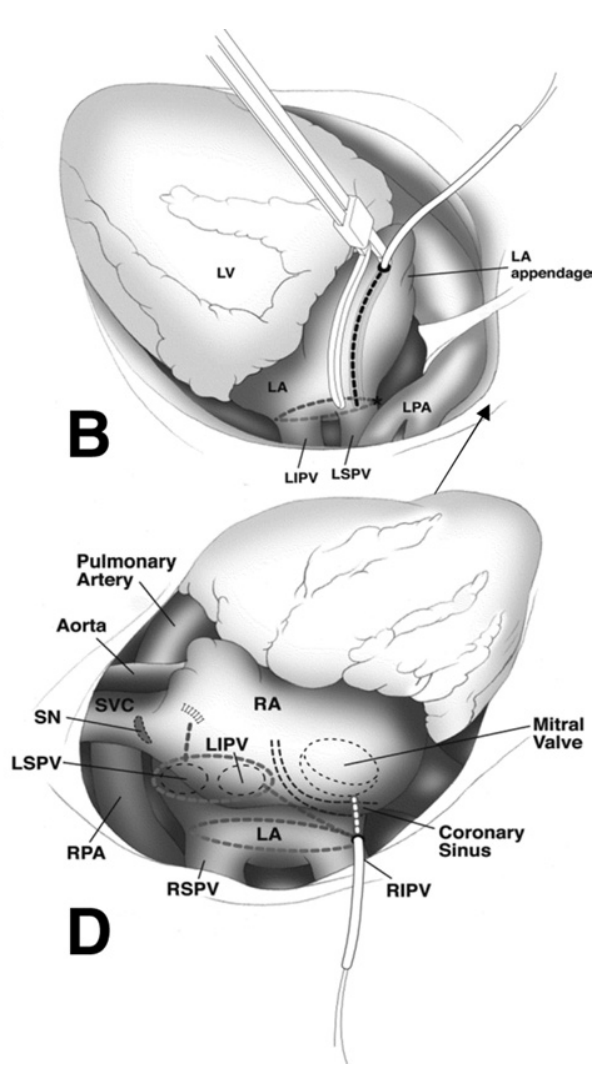

Figure 2. Left atrial lesion set. SVC, Superior vena cava; $S N$, sinus node; $R S P V$, right superior pulmonary vein; $R I P V$, right inferior pulmonary vein; $I V C$, inferior vena cava; $R P A$, right pulmonary artery; $L P A$, left pulmonary artery; $L A$, left atrium; $L S P V$, left superior pulmonary vein; LIPV, left inferior pulmonary vein. 
Table 2. Heart rhythm at follow-up

\begin{tabular}{|c|c|c|c|c|c|c|c|}
\hline Patient no. & Operation & $3 \mathrm{mo}$ & $6 \mathrm{mo}$ & $9 \mathrm{mo}$ & $12 \mathrm{mo}$ & $18 \mathrm{mo}$ & $24 \mathrm{mo}$ \\
\hline 1 & Maze + CAB & $\mathrm{NSR}^{*}$ & NSR $\ddagger$ & $\mathrm{NSR}^{*}$ & $\mathrm{NSR}^{*}$ & NSR $\|$ & NSR $\|$ \\
\hline 2 & Maze + CAB & $\mathrm{NSR}^{*}$ & NSR $\|$ & $\mathrm{NSR}^{*}$ & $\mathrm{NSR}^{*}$ & NSRף & - \\
\hline 3 & Maze + CAB & $\mathrm{NSR}^{*}$ & $\mathrm{AF} \|$ & No data & $\mathrm{NSR}^{*}$ & $\mathrm{NSR}^{*}$ & - \\
\hline 4 & Maze + AVR & $\mathrm{NSR}^{*}$ & NSR $\|$ & No data & NSR\# & Expired & - \\
\hline 5 & Maze + CAB & NSR & NSR\| & NSR† & AFL† & NSR† & - \\
\hline 6 & Maze & NSR§ & NSR§ & NSR\| & NSR\| & $\mathrm{NSR}^{*}$ & - \\
\hline 7 & Maze + CAB & NSR & NSR & NSRt & NSR\| & - & - \\
\hline 8 & Maze + CAB & NSR\| & $\mathrm{NSR}^{*}$ & $\mathrm{NSR}^{*}$ & $\mathrm{NSR}^{*}$ & - & - \\
\hline 9 & Maze + CAB & No data & $\mathrm{NSR}^{*}$ & - & - & - & - \\
\hline 10 & Maze + AVR & NSR & NSR & NSR $\dagger$ & NSRף & - & - \\
\hline 11 & Maze + CAB & $\mathrm{NSR}^{*}$ & - & - & - & - & - \\
\hline 12 & Maze + CAB & NSR川 & - & - & - & - & - \\
\hline
\end{tabular}

$C A B$, Coronary artery bypass; $N S R$, normal sinus rhythm; -, end of follow-up; $A F$, atrial fibrillation; $A F L$, atrial flutter; $A V R$, aortic valve replacement. *Electrocardiographic strip. †Pacemaker interrogation. ¥Twenty-four-hour Holter monitoring. §Event monitor. ||Self-report. ILong-term monitoring, CARDIONET (120-hour Holter monitoring). \#Long-term monitoring with hospital telemetry for more than a month.

the umbilical tape around them, the bipolar device is placed around the left pulmonary veins, and the ablation then takes place (Figure $2, B$, black arrow). The crossclamp is applied to the ascending aorta, and myocardial arrest is induced by using cold blood cardioplegic solution.

A purse-string suture is then placed on the tip of the left atrial appendage, and a bipolar radiofrequency device is inserted inside the atrial appendage, with its tip placed into the orifice of the left superior pulmonary vein to create a linear lesion (Figure $2, B$ ). The device is then withdrawn, and the left atrial appendage is excluded from the rest of the left atrium by stapling or suturing the base of the appendage from the outside. In the last 4 cases of this series, instead of using a purse-string suture and an application of the bipolar radiofrequency device endocardially and epicardially, a simple epicardial ablation method was used to connect the base of the left atrial appendage to the left pulmonary veins. This was achieved by clamping the base of the left atrial appendage together with the antral portion of the left superior pulmonary vein with the bipolar device, so the ablation line is connected or overlapped, with the left pulmonary veins encircling the lesion.

The last purse-string suture is placed to the inferior aspect of the right inferior pulmonary vein. The bipolar radiofrequency device is then placed so that the upper jaw is within the left atrium (endocardially) and the bottom one is pointed to the left and underneath the IVC and across the base of the heart. The ventricular apex is then retracted in a cephalad direction out of the pericardium by using the left hand to expose the intrapericardial segments of both left pulmonary veins. This maneuver allows the surgeon to place the epicardial (bottom) jaw of the bipolar device right to the tip of the left inferior pulmonary vein. After the correct placement of the device, an ablation is applied, resulting in a connecting lesion between the right and left inferior pulmonary veins (Figure 2,C). The same purse-string suture is used to create a lesion from the inferior pulmonary veins connecting the lesion down to the posterior mitral valve annulus. A linear cryoprobe is placed across the bottom of the left atrium and toward the annulus of the mitral valve. An important landmark to assist in the correct placement of the cryoprobe is the retrograde coronary sinus cath- eter placed in the coronary sinus. The cryoprobe is palpated across the coronary sinus catheter, allowing at least $1 \mathrm{~cm}$ of the cryoprobe to be placed beyond the coronary sinus and toward the mitral valve annulus. Completion of the ablation of the coronary sinus is done by using the $15-\mathrm{mm}$ right angle probe placed epicardially over the coronary sinus and directly related to the placement of the previous lesion (Figure 2,D). The cryoprobe is then retracted from the left atrium, and the last purse-string suture is tied. This concludes the maze procedure, and the combined procedure of a CABG or AVR is then performed.

\section{Results}

There were no perioperative deaths or cerebrovascular accidents/transient ischemic attacks that were documented in this group of patients. No other major complications were documented; 1 patient was readmitted to the intensive care unit because of fluid retention. An automatic implantable cardioverter defibrillator pacemaker was implanted in 1 patient because of a preoperative left ventricular ejection fraction of $20 \%$, along with a positive postoperative electrophysiologic study result. Two patients required a dualchamber pacemaker implantation because of sinus node dysfunction, 1 of which was documented preoperatively. The mean hospital stay was $8.3 \pm 2.9$ days. In a mean follow-up of $13 \pm 6.04$ months (median, 12 months; range, 3-24 months), there was 1 late death, and no late thromboembolic events were documented. The late death was documented in a 72-year-old patient 15 months after the original operation involving an AVR and a maze procedure. The patient had a repeat operation 12 months after the first one because of early failure of the aortic tissue valve that was implanted. After the second operation, he had perioperative atrial fibrillation, which was resolved after treatment. He was discharged to a nursing home and died 3 months after the repeat operation because of end-stage respiratory failure. 
All other patients completed follow-up, including a qualityof-life assessment. All but one are in sinus rhythm. This patient had a combined maze procedure and CABG and short bursts of atrial flutter after his 9-month postsurgical follow-up. He had undergone what seems to be a successful catheter ablation 11 months after the operation and is currently in sinus rhythm.

Table 2 describes the cardiac rhythm at each follow-up period. At 3 months, 11 (92\%) patients were available for follow-up; at 6 months, 10 patients completed the follow-up $(100 \%)$. Seventy-seven percent of the patients were available for follow-up at 9 months, with the 2 missing patients available for follow-up at a later time point. All 9 patients were available for follow-up at 1 year; 5 patients were available for follow-up at 18 months (100\%). At last followup, none of the patients were taking a class I or III antiarrhythmic drug, and no calcium-channel blockers were reported. In 9 patients $\beta$-blockers were used, 8 cases of which were in patients after coronary bypass surgery and 1 after AVR. In none of the patients were $\beta$-blockers subscribed for arrhythmia control. The mode in which we captured the rhythm is well documented in Table 2 . There were 4 methods of long-term monitoring mentioned: event monitoring, pacemaker or automatic implantable cardioverter defibrillator interrogation, 1-week Holter monitoring with the Cardionet system, and continuous telemetry monitoring during readmission to the hospital. There were 43 time points at which rhythm was captured and then reported; in 17 (40\%) time points rhythm verification was based on means other than electrocardiographic strip or self-reporting (Table 2). The rhythm status of 2 patients with a follow-up of longer than 6 months is not based on any sort of long-term monitoring or 24-hour Holter monitoring; however, in both patients electrocardiographic strips from at least 3 different time points were obtained to support their rhythm status.

In 5 additional patients (maze procedure combined with AVR in 3 patients and with CABG in 2 patients), a conversion of the original surgical plan occurred. In these patients the application of a reliable lesion across the mitral valve isthmus was impossible. Therefore a very small vertical atriotomy was performed to allow direct vision and precise application of the lesion. The rest of the procedure was performed with multiple purse-string sutures, as previously described. All patients in this group had an uneventful postoperative course, with 1 patient having relapses of atrial flutter late in the follow-up. This patient is now a candidate for an electrophysiologic study and possible catheter ablation.

\section{Discussion}

In recent years we have witnessed a significant increase in the number of surgical procedures performed to ablate atrial fibrillation. During the same period, a variety of surgical ablation devices have been introduced and used by cardiac surgeons. ${ }^{9}$ Currently, it is very difficult to assess the relative success of such procedures when compared with the original maze procedure. This is because much of the published data deal with different ablation devices and a different lesion set. The original maze procedure is still considered by many to be the gold standard for the surgical treatment of atrial fibrillation ${ }^{1,2}$; therefore when designing this new procedure, the goal was to maintain the basic principles of the maze procedure with regard to the lesion set. However, instead of using the cut-and-sew approach, the lesions were applied by using 2 different ablation technologies in which we gained a significant amount of experience in the past few years. This new surgical approach was introduced in a very careful way. During the period in which this surgical series was established, more than 150 patients underwent operations for atrial fibrillation in our institute; however, only 25 patients underwent operations for a combination of a maze procedure and either CABG or AVR. No specific guidelines were used to include patients in this series, and therefore not all the potential patients underwent operations with the technique. We allowed a significant time for follow-up to assess the safety and efficacy of the new approach before recommending using it within the appropriate indications.

Using this technique allows surgeons to isolate the pulmonary veins before the initiation of cardiopulmonary bypass. Although ablation of the right pulmonary veins is relatively simple, beating-heart ablation of the left pulmonary veins is somewhat challenging and should be approached with caution. Hemodynamic stability should be maintained while manipulating the left ventricular apex, and dissection around the left pulmonary veins should be done carefully because they might be much distended while the left ventricular apex is shifted.

Another important aspect of bipolar radiofrequency pulmonary vein isolation is the testing of a conduction block. One of the potential limitations of this series is that we never tested the pulmonary veins for a conduction block, and the technique was based on multiple repeated ablations (4-5 applications on each side) and assumed transmurality. Future cases are going to be performed with the appropriate testing for conduction block.

When performing the cryolesions on a beating heart, the endocardial application can be performed safely and effectively because the cryoprobe is adhered to the endocardium, and direct cooling is then applied, with the ice ball forming on the epicardial surface serving as an indicator for transmurality. This is our routine approach for right-sided ablation unless the right atrium should be approached for a tricuspid valve procedure or atrial septal defect closure. ${ }^{4}$

The early results from the procedure demonstrate that it is safe and effective in this small series. It is clear to us that the current technology available for surgeons is limited and 
would not allow us to apply this new approach in all cases with indications if we are to maintain all the basic principles of the maze procedure. In $5(30 \%)$ patients of this series, the procedure was modified, and a small left atrial atriotomy was applied to ensure a reliable lesion across the left atrial isthmus, one of the most significant lesions of the maze procedure. ${ }^{10}$

There were 2 cases of atrial flutter documented during the follow-up, one in a patient in whom the procedure was performed with the multi-purse string approach (without small left atrial atriotomy). The patient was known to have ischemic cardiomyopathy and a low ejection fraction (20\%). He had the atrial flutter late in the follow-up and was treated successfully with catheter ablation. Because the ablation was done in an out-of-state medical institution, we do not have any data regarding the electrophysiologic study. Regardless, this patient's condition makes an excellent case for a potential limitation of the new technique, which is the creation of a reliable ablation line across the mitral valve isthmus. One possible solution for the problem is new, specially designed ablation devices to address this problem. On the contrary, in the second patient, who was shown to have short bursts of atrial flutter, the mitral valve isthmus lesion was created by using a small atriotomy, and therefore the probe placement and the reliability of the mitral valve isthmus ablation is not questionable. Because the patient's symptoms are well controlled with amiodarone, no electrophysiologic study is scheduled, and it will be performed only if relapses of supraventricular arrhythmias are documented after the discontinuation of the antiarrhythmic drug.

Based on the results presented here, it seems that there is a place to include the procedure as part of our treatment options for patients with atrial fibrillation, especially when aortic valve surgery and $\mathrm{CABG}$ are indicated. However, at this time, using this technique on a beating heart should be approached very carefully. It is clearly indicated that technically this new approach can be performed on a beating heart; however, there are some safety concerns, mainly with regard to the increased risk of thromboembolic events. This might be directly related to air that could be introduced to the left atrium during the placement of the ablation devices in the left atrium and to the potential of char forming lesions, especially when dealing with bipolar radiofrequency devices in a bloody environment. ${ }^{11,12}$ Future research should be focused on the detection of thromboembolic events, especially when applying endocardial ablation to a beating heart.
Our limited experience with this approach also suggests that, currently, none of the devices available in the market are perfect for applying the lesion across the mitral valve isthmus, and based on the importance of the lesion, surgeons should be prepared to modify their approach when necessary to ensure a reliable lesion set.

The results reported here should be approached with caution. The major limitation of this series is its small size and the relatively short follow-up.

We thank Drs Burton, Collazo, Lefrak, Massimiano, Rongione, and Speir for their support.

The artwork for the figures was provided by Atricure, Cincinnati, Ohio.

\section{References}

1. Barnett SD, Ad N. Surgical ablation as the treatment for the elimination of atrial fibrillation: a meta-analysis. $J$ Thorac Cardiovasc Surg. 2006;131:1029-35.

2. Prasad SM, Maniar HS, Camillo CJ, Schuessler RB, Boineau JP, Sundt TM, et al. The Cox maze III procedure for atrial fibrillation: long-term efficacy in patients undergoing lone versus concomitant procedures J Thorac Cardiovasc Surg. 2003;126:1822-8.

3. Cox JL, Schuessler RB, Boineau JP. The development of the Maze procedure for the treatment of atrial fibrillation. Semin Thorac Cardiovasc Surg. 2000;12:2-14.

4. Ad N, Cox JL. The minimally invasive maze procedure. J Card Surg. 2004;19:196-200.

5. Damiano RJ Jr. Alternative energy sources for atrial ablation: judging the new technology. Ann Thorac Surg. 2003;75:329-30.

6. Viola N, Williams MR, Oz MC, Ad N. The technology in use for the surgical ablation of atrial fibrillation. Semin Thorac Cardiovasc Surg. 2002;14:198-205.

7. Gaynor SL, Schuessler RB, Bailey MS, Ishii Y, Boineau JP, Gleva MJ, et al. Surgical treatment of atrial fibrillation: predictors of late recurrence. J Thorac Cardiovasc Surg. 2005;129:104-11.

8. Holman WL, Ikeshita M, Douglas JM Jr, Smith PK, Cox JL. Cardiac cryosurgery: effects of myocardial temperature on cryolesion size. Surgery. 1983;93:268-72.

9. Bakir I, Casselman FP, Brugada P, Geelen P, Wellens F, Degrieck I, et al. Current strategies in the surgical treatment of atrial fibrillation: review of the literature and Onze Lieve Vrouw Clinic's strategy. Ann Thorac Surg. 2007;83:331-40.

10. Cox JL, Ad N. The importance of cryoablation of the coronary sinus during the Maze procedure. Semin Thorac Cardiovasc Surg. 2000;12: 20-4.

11. McGreevy KS, Hummel JP, Jiangang Z, Haines DE. Comparison of a saline irrigated cooled-tip catheter to large electrode catheters with single and multiple temperature sensors for creation of large radiofrequency lesions. J Interv Card Electrophysiol. 2005;14: $139-45$.

12. Wazni OM, Rossillo A, Marrouche NF, Saad EB, Martin DO, Bhargava $M$, et al. Embolic events and char formation during pulmonary vein isolation in patients with atrial fibrillation: impact of different anticoagulation regimens and importance of intracardiac echo imaging. J Cardiovasc Electrophysiol. 2005;16:576-81. 